\title{
Measurement of Intramyocardial Phasic Blood Flow Using Impedance Plethysmography
}

\author{
Muneyuki Horikawa, M.D.
}

\begin{abstract}
SUMMARY
Impedance plethysmography was applied for the measurement of intramyocardial phasic blood flow. In the region of the left circumflex. artery M-shaped blood flow curve was obtained in the outer and middle layer. This pattern did not resemble that of the inner layer. At the time of reactive hyperemia, increase in blood volume was seen within the myocardium.
\end{abstract}

\section{Additional Indexing Words :}

Reactive hyperemia Specific resistance Urethane coated electrode

COMPLETE method of measurement of intramyocardial phasic blood flow 4 has never been settled up, though some aimed at it by indirect method in dog experiments. ${ }^{1)}$ Although $\mathrm{N}_{2} \mathrm{O}$ method, ${ }^{2)}$ isotope clearance method using ${ }^{86} \mathrm{Rb}^{3)}$ or ${ }^{131}{ }^{41}$ and indicator dilution method $^{5 /}$ might give information on coronary flow, they are not informative on its phasic change. On the other hand, an electromagnetic flowmeter can measurc phasic coronary flow, but it is limited to measure blood flow of comparatively large coronary artery exposed on the surface of the heart. In this study, in order to measure intramyocardial phasic blood flow, the impedance plethysmography with divised electrodes was applied.

\section{Principles and Methods}

The impedance $\left(\mathrm{Zc}_{\mathrm{c}}\right)$ in ohm of a rectangular myocardial segment is determined by its specific resistance $\left(\rho_{c}\right)$ expressed in ohm centimenter and its dimensions, length $(l)$ in centimeter, and cross section (s) in square centimeter, according to the following formula $:^{(0), 3)}$

$$
\mathrm{Zc}=\rho_{\mathrm{c}} \cdot \frac{l}{\mathrm{~s}}
$$

Since any fraction remains unchanged if multiplied or divided by a value which equals one, equation (1) may be expressed as:

From the Department of Internal Medicine, School of Medicine, Keio University, 35 Shinanomachi, Shinjuku-ku, Tokyo 160, Japan.

Received for publication February 14, 1974. 


$$
\mathrm{Zc}=\rho_{\mathrm{c}} \cdot \frac{l}{\mathrm{~s}} \cdot \frac{l}{l}
$$

As the product $\mathrm{s} \cdot \mathrm{l}$ is equal to volume (Vc), equation (2) may be rewritten as:

$$
\mathrm{Z}_{\mathrm{c}}=\rho_{\mathrm{e}} \cdot \frac{l^{2}}{\mathrm{~V}_{\mathrm{c}}}
$$

If the length of the rectangular myocardial segment is constant and the volume is increased by the change in blood volume $(\triangle \mathrm{Vb})$, likewise the impedance of only increased blood volume $(\mathrm{Zb})$ becomes as follows:

$$
\mathrm{Zb}=\rho_{\mathrm{b}} \cdot \frac{l^{2}}{\Delta \mathrm{Vb}}
$$

where $\rho_{\mathrm{b}}$ equals the specific resistance of blood.

When $\mathrm{Zb}$ acts in parallel anatomically and electrically as a parallel impedance to $\mathrm{Zc}$, the change in impedance of $\mathrm{Zc}_{\mathrm{c}}\left(\Delta \mathrm{Z}_{\mathrm{c}}\right)$ is written as follows as a result of the increased blood volume:

$$
J Z_{c}=\frac{1}{1 / Z b+1 / Z c}-Z_{c}=-\frac{Z c^{2}}{Z b+Z c}
$$

and furthermore $\mathrm{Zb}$ is much greater than $\mathrm{Zc}$, therefore $J \mathrm{Zc}$ is approximately given by :

$$
\lrcorner \mathrm{Zc}=-\frac{\mathrm{Zc}^{2}}{\mathrm{Zb}+\mathrm{Zc}} \simeq-\frac{\mathrm{Zc}^{2}}{\mathrm{Zb}}
$$

By substituting eauation (3) and (4) into equation (6), equation (6) is rearranged as:

$$
\frac{\Delta \mathrm{Vb}}{\mathrm{Vc}}=-\frac{\rho_{\mathrm{b}}}{\rho_{\mathrm{c}}} \cdot \frac{\Delta \mathrm{Zc}}{\mathrm{Zc}}
$$

Equation (7) means that the product of the fractional specific resistance of blood to cardiac muscle multiplied by the fractional change in impedance, approximately equals the fractional change in blood volume within the segment. The negative sign on the right side of equation (7) indicates that a small increase in volume brings about a decrease in electrical impedance. An increase or decrease of impedance is therefore produced by the blood being reduced or added to the segment respectively. Thus, if the fractional specific resistance of blood to cardiac muscle and the fractional change of $Z_{c}$ are measurable, one should be able to calculate the fractional blood volume per unit mass of the myocardium.

Ten mongrel dogs weighing 16 to $21 \mathrm{Kg}$ were subjected to the series of experiments. They were anesthetized with sodium pentobarbital ( 25 to $50 \mathrm{mg} / \mathrm{Kg}$, iv) and the chest was opened in the 4th left intercostal space under artificial respiration. The heart was exposed by making longitudinal incision through the pericardium to approach the left coronary artery and the free wall of the left ventricle. A diagram of set-up of apparatus and preparation is shown in Fig. 1. An electromagnetic flowmeter probe (Nihon Kohden Kogyo Co, Tokyo, Japan) was placed 


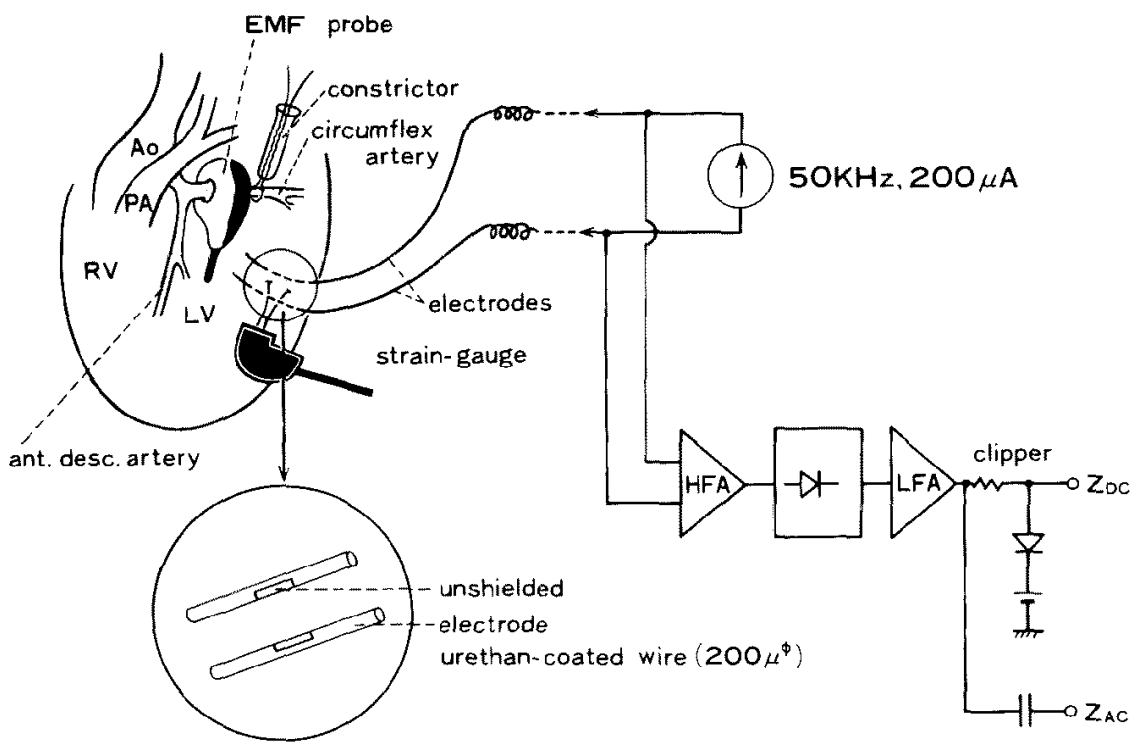

Fig. 1. Schematic representation of the apparatus for the measurement of intramyocardial phasic blood flow. EMF, elcctromagnctic flowmeter; HFA, high frequency amplifier; LFA, low frequency amplifier.

on the left circumflex artery to measure the coronary flow and close to the distal side of the probe a constrictor was placed around the coronary artery to occlude the coronary flow for several seconds. A catheter connected to a pressure transducer (Nihon Kohden) was inserted into the left ventricle via the left atrium to measure left ventricular pressure. A pair of urethane coated electrodes ( $200 \mu$ in diameter) which had small uncoated parts $(5 \mathrm{~mm} \times 1 / 3$ of circumference) were inserted face to face at the site of measurement. Each uncoated part was placed approximately $8 \mathrm{~mm}$ apart at the same depth. Near the electrodes a strain-gauge was located for observing a change in myocardial segment length around the electrodes before and during occlusion. A block diagram of the impedance plethysmograph (Nihon Kohden), whose frequency characteristics is DC to $100 \mathrm{~Hz}$, is shown in Fig. 1. Between the paired electrodes a $50 \mathrm{kHz}, 200$ microampere constant current was supplied and impedance changes were continuously detected as voltage changes with a high-frequency amplifier. Detected impedance changes after dealing with a rectifier and low-frequency amplifier circuit were divided into 2 kinds of signals, one is DC component $\left(Z_{\mathrm{DC}}\right)$ which is limited above some reference level by a clipping circuit and the other is the impedance consisting of $\mathrm{AC}$ component $\left(\mathrm{Z}_{\mathrm{AC}}\right)$.

After the recording a control state, the impedance was recorded during several second occlusion of the left circumflex artery (Nihon Kohden, Polygraph RM1000). Fractional change in impedance was obtained by dividing the maximum difference of $\mathrm{Z}_{\mathrm{DC}}$ before and during occlusion $(\Delta \mathrm{Z})$, by the impedance at enddiastolic phase before occlusion $\left(Z_{E D}\right)$. Changes in impedance caused only by cardiac movement is canceled by the procedure of subtracting the impedance during occlusion from that before occlusion, providing that the heart has the samc movement before and during occlusion. After the foregoing procedures were over, the 
specific resistance of blood and cardiac muscle was measured by the same impendance plethysmograph in the free wall of the left ventricle and blood contained in the left ventricular cavity respectively, immediately after induced ventricular fibrillation. The depth of electrodes was measured in a manner perpendicular to the epicardium with cutting the ventricular free wall as close as possible to the electrodes and designated as a relative depth to wall thickness.

\section{Results}

Fig. 2 shows one of the recordings illustrating a method for obtaining intramyocardial phasic blood flow depicted on the bottom tracing. In this case a pair of electrodes was inserted into the inner layer at depth of $8 \mathrm{~mm}$ from the epicardium (wall thickness $10 \mathrm{~mm}$ ). In $2 \mathrm{sec}$ after occluding the left circumflex artery, $Z_{\mathrm{ED}}$ was increased from 590 to $630 \mathrm{ohms}$ as a result of the reduction of blood and at that time $Z_{\mathrm{AC}}$ changed from a control state. The left ventricular pressure and the segment length remain approximately unchanged. The postmortem fractional specific resistance of blood to

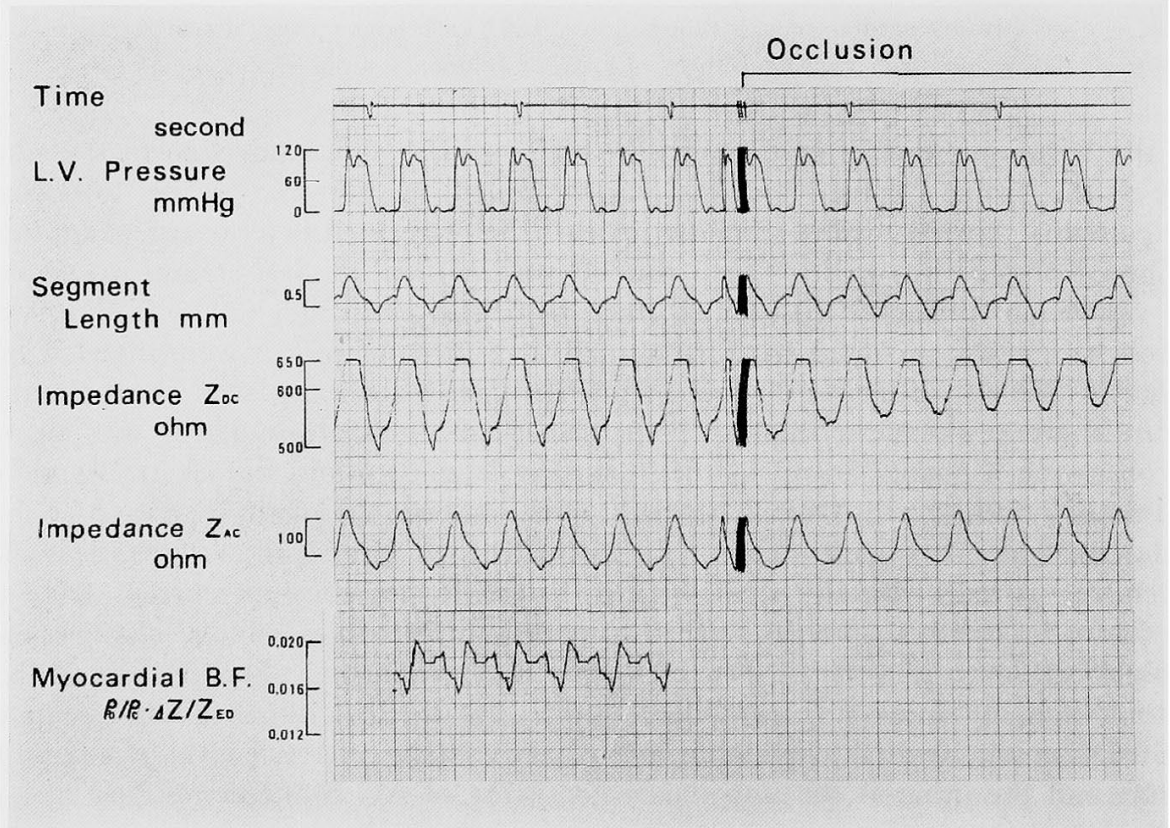

Fig. 2. Representative recording showing method for $_{\mathrm{s}}$ obtaining intramyocardial phasic blood flow. Relative myocardial depth 0.8 . From top to bottom; time scale, left ventricular pressure, segment length, impedance $\mathrm{Z}_{\mathrm{DC}}$, impedance $\mathrm{Z}_{\mathrm{AC}}$, and measured intramyocardial phasic blood flow (a solid line connecting the values successively). $\mathrm{Z}_{\mathrm{DC}}$ was increased about $40 \Omega$ at end-diastolic phase $2 \mathrm{sec}$ after occluding the left circumflex artery. $Z_{A C}$ changed from a control state. L. V. pressure and segment length remain approximately unchanged. 
cardiac muscle was measured 0.29 . Thus, the product of the fractional specific resistance 0.29 multiplied by the whole quantity the difference of $Z_{D C}$ at each equivalent point of a cardiac cycle before and during occlusion divided by $Z_{\mathrm{ED}}(590 \mathrm{ohms})$, leads to the intramyocardial phasic blood flow contours as depicted on the bottom tracing in Fig. 2.

In a manner as stated above, observations were performed in the region of the left circumflex artery in each layer. The results are shown in Fig. 3 illustrating the average blood flow at the same depth of the myocardium. The numerals denoted by $d$, express the average depth of 2 electrodes in pair. When the depth of the 2 electrodes in pair differed more than $2.5 \mathrm{~mm}$, the data were excluded out of evaluation. In the outer and middle layer, M-shaped blood flow curve was obtained. The first peak was coincident with isovolumetric contraction period and the second one was located in diastole.

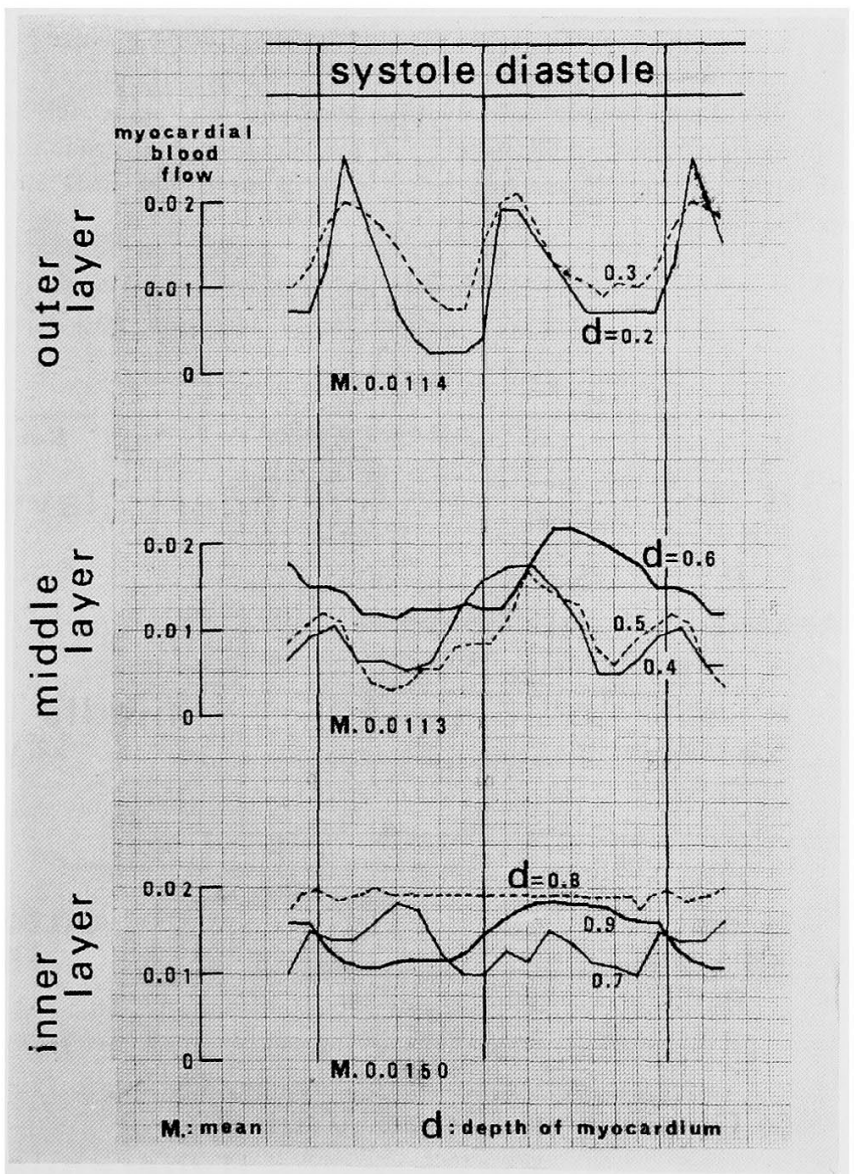

Fig. 3. Intramyocardial blood flow curves in the outer, middle and inner layers. In the outer and middlc layer, M-shaped blood flow curve was seen, while that in the inner layer was not clear M-shaped. 


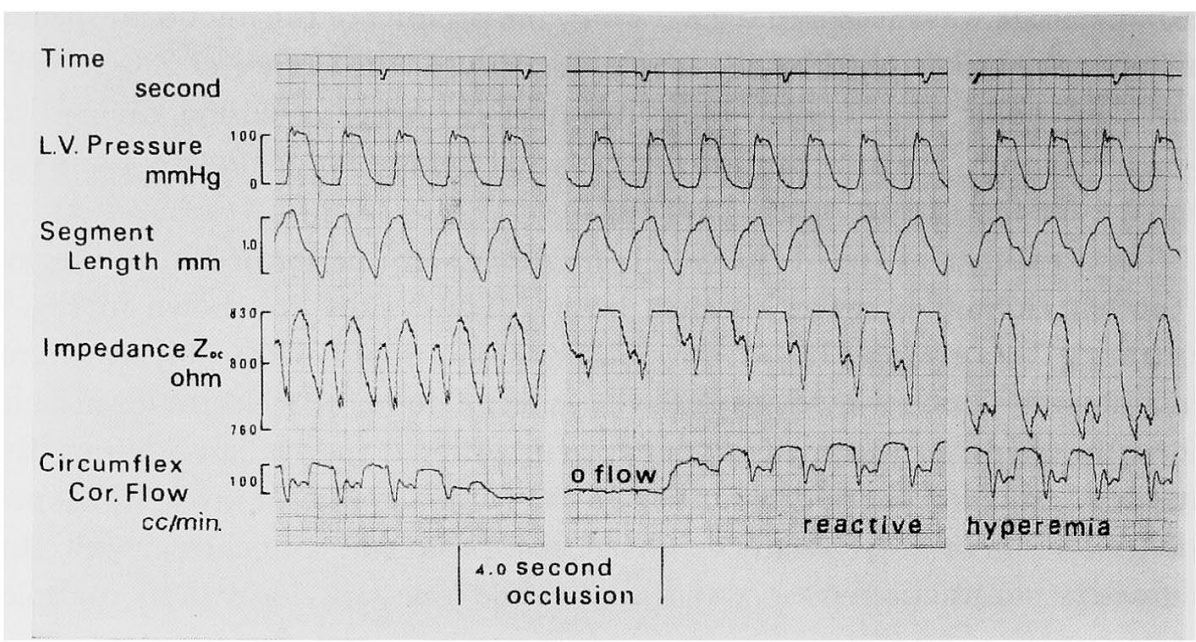

Fig. 4. Cumparison of increase in blood volume during reactive hyperemia between the electromagnetic flowmeter and the impedance method. Relative myocardial depth 0.4 . During occlusion $Z_{D C}$ increased, while EMF showed zero flow. Following a 4.0 sec occlusion, $\mathrm{Z}_{\mathrm{DC}}$ gradually decreased down and finally reached below control level, and EMF showed reactive hyperemia.

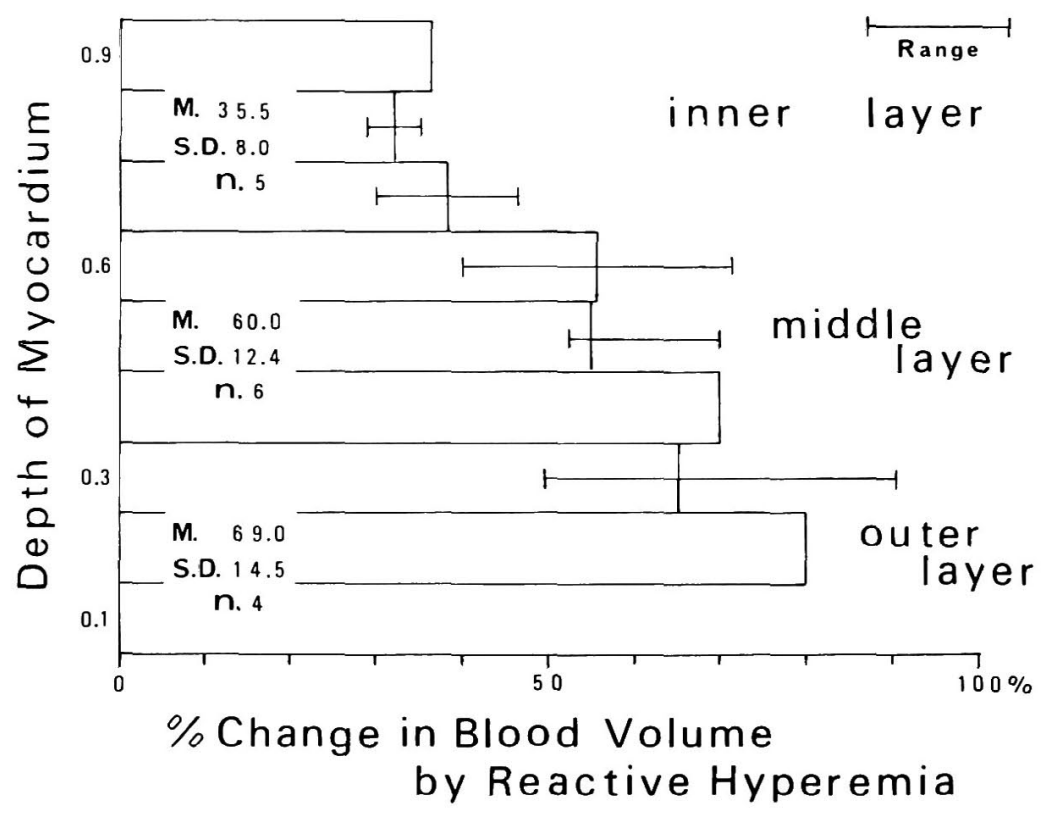

Fig. 5. Percent ratio of increase in blood volume at the time of reactive hyperemia in each layer. Discussed in text. 


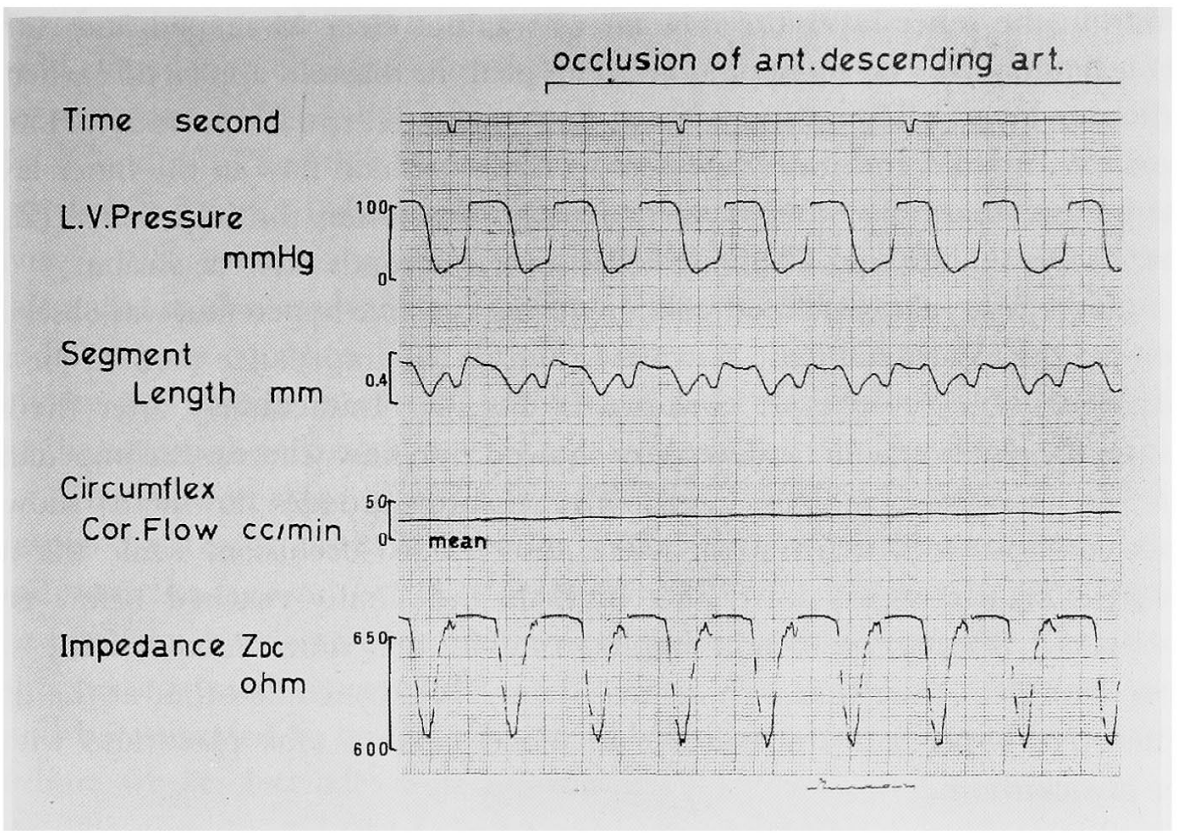

Fig. 6. Effect of occlusion of the anterior descending artery on flow in the region of the left circumflex artery. During occlusion, EMF mean flow on the left circumflex artery slightly increased and coincidentally small decrease in impedance was seen.

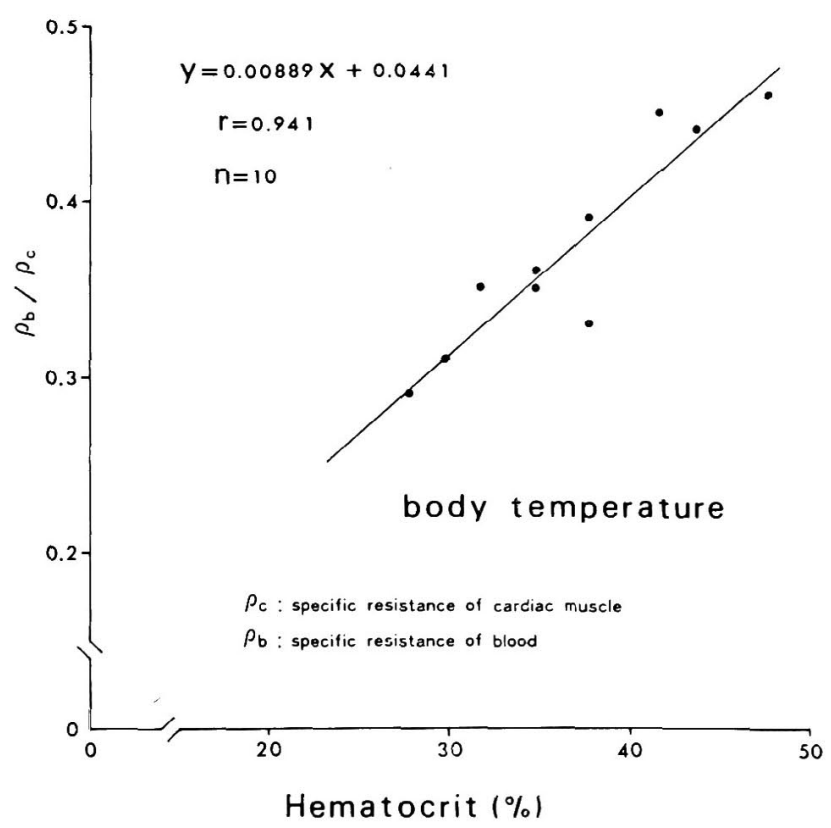

Fig. 7. Linear correlation between ratio of the specific resistance of blood to cardiac muscle and hematocrit. 
While in the inner layer the flow curve was not clear M-shaped and rather has a tendency to be monophasic. The peak in diastole appeared earlier in the outer layer. The average blood flow was $0.0114,0.0113$, and 0.0150 in the outer, middle and inner layer respectively. Blood flow in the inner layer was greater than any other layers. In all observations the impedance $\left(Z_{D C}\right)$ was always maintained at higher level several seconds after occlusion.

Increased values of blood volume during reactive hyperemia was observed both by the electromagnetic flowmeter and in the impedance method for the purpose of comparison. It is shown in Fig. 4. Immediately after the occlusion the electromagnetic flowmeter showed zero flow whereas the impedance $\mathrm{Z}_{\mathrm{DC}}$ was increased a little later. The electromagnetic flowmeter showed reactive hyperemia which took place after $4.0 \mathrm{sec}$ occlusion, while the impedance $\mathrm{Z}_{\mathrm{DC}}$ decreased down little by little and finally reached below control level. This phenomenon implies that at this time blood volume was more abundant than that in a control state. It is concluded that at the time of reactive hyperemia the increase in blood volume took place also within the myocardium.

Ratio of increased blood volume to fractional blood volume at the time of reactive hyperemia are shown in percentage in Fig. 5. Average values of percent change in blood volume in middle and outer layer are $60.0 \%$ (s.d. 12.4 ) and $69.0 \%$ (s.d. 14.5) respectively, while that in the inner layer is $35.5 \%$ (s.d. 8.0). This may indicate that the capacity of the coronary vascular reserve is poor in the inner layer in comparison with that in the outer layer.

Fig. 6 shows the effect of occlusion of the anterior descending artery on the flow in the region of the left circumflex artery. When the anterior descending artery was occluded, the electromagnetic mean flow on the left circumflex artery slightly increased and coincidentally small decrease in impedance was seen as a result of increase in blood volume in the region of the left circumflex artery. Similar observation, an increase in coronary blood flow in vessels adjacent to the occluded arteries, was made by Herzberg et al. ${ }^{8}$

The ratio of the specific resistance of blood to that of cardiac muscle is plotted as a function of hematocrit in Fig. 7. A significant correlation $(r=$ 0.941) was found between them. The linear regression equation for $\rho_{\mathrm{b}} / \rho_{\mathrm{c}}$ as a function of hematocrit was calculated to be $\mathrm{Y}=0.00889 \mathrm{X}+0.0441$.

\section{Discussion}

The fundamental equation already mentioned in the foregoing chapter becomes available only when the movement of the myocardium at the site of electrodes should have the same way before and during occlusion. If it is 
altered, some errors might intervene the results because it is impossible to avoid impedance changes caused by cardiac movement. Relative errors of the impedance $(Z)$ on the left side in equation ( 1 ), equals the sum of those of $\rho, l$, and $\mathrm{s}$. Therefore, suppose the distance between the paired electrodes is settled at $8 \mathrm{~mm}, \pm 0.8 \mathrm{~mm}$ change in cardiac movement caused by occlusion gives $\pm 10 \%$ relative errors of $\mathrm{Z}$ under the condition of constant $\rho$ and $\mathrm{s}$. This value is also equivalent to relative errors of fractional blood volume on the left side in equation (7). In all observations of this study, errors caused only by the change in cardiac movement before and during occlusion were less than several percents.

The surface areas of electrodes used in this experiment were small as compared with the distance between paired electrodes, therefore under these conditions the current fiux distribution would be curved outside of a straight line between the electrodes. But it is supposed that the current flows dominantly in the straight course between the electrodes, so the experimental results could be estimated to be the changes only in the localized parts of the myocardium where electrodes werc inserted.

The absolute values of the impedance obtained in this series of experiments are smaller than the values calculated theoretically by substituting the known numerals into the formula $\mathrm{Z}=0 \cdot \mathrm{l} / \mathrm{s}$. This must be due to the traumatic bleeding caused by the insertion of the electrodes. By this traumatic injury, shortening of the distance between paired electrodes and enlargement of the effective surface area of the electrodes took place. Accordingly, the former gave smaller value to $l$ and the latter gave larger one to s than those expected theoretically.

Thinking over the electrical impedance in biological materials, behavior of tissue and fluid in a time-varying field should at first be taken into consideration. Referring to Kraus' criteria ${ }^{9}$ for classification whether the medium behaves as a conductive, semiconductive or dielectric, sea water can be substitutive for cardiac muscle. Therefore, the following speculation is to be done on sea water in stead of cardiac muscle. Here cardiac muscle is thought to be almost perfect conductor at $50 \mathrm{kHz}$. It is concluded that the change in impedance measured in this experiment was produced essentially by a resistive change.

When the left circumflex artery is occluded, it is estimated that all blood contained in the arterioles, capillaries, and venules is squeezed into the venous side by myocardial contraction. Therefore, the intramyocardial blood flow obtained in this experiment can be thought to be total blood flow in situ. However, this is pretty lower than that of Gibson et al. ${ }^{10)}$ They used isotope techniques in order to determine the blood volume in coronary arterioles, 
capillaries, and venules in cardiac arrest, and obtained $6.6 \mathrm{ml} / 100 \mathrm{Gm}$ cardiac muscle in average (fractional blood volume; 0.066 , if the specific gravity of cardiac muscle is 1.0 ). The reason why the mean values in this method was considerably different from the data of Gibson et al ${ }^{103}$ might probably due to the fact that blood contained in the coronary vessels was not completely squeezed out.

The peak which appeared in isovolumetric contraction period is coincident with so called early systolic backflow observed in the electromagnetic flowmeter tracing of the left circumflex artery. The impedance plethysmography cannot differentiate forward flow from backward. Therefore, the first peak appeared inversely to that of the electromagnetic flowmeter.

The second peak appearing in the outer and middle layer must be identical with the forward larger flow in diastole observed in the electromagnetic flowmeter on the left circumflex artery. In the middle layer the second peak was seen later than that in the outer layer. This mechanism is not clear, but it might be due mainly to the difference of the intramyocardial pressure.

It has been pointed out that myocardial blood flow in the endocardial side of the left ventricle is larger than that in the epicardial side.41,11,12) In this study mean flow in the inner layer was larger than that in other layers. Myers and Honig ${ }^{13)}$ studied number of intramyocardial capillaries and its distribution, and concluded that a vascularity gradient increasing from epicardium to endocardium exists throughout the ventricular myocardium.

The monophasic tendency of phasic flow in the inner layer could be explained by the anatomical characteristics of the inner layer of the left ventricle. Some investigator( ${ }^{14)-16)}$ have found that intramyocardial tissue pressure in the endocardial side is much larger than that in the epicardial side during systole and even exceeds left ventricular pressure. Accordingly, the pressure condition surrounding the vessels in the inner layer must fluctuate in a wide range. It is obvious that there should be a wide fluctuations in the blood flow in a same way. In this view there is some conflict in findings in Fig. 3 in which the flow curve in the inner layer tends to be monophasic. The inner layer of the left ventricle has some anatomical drainage to the ventricular cavity. ${ }^{17)}$ Therefore, it can be supposed that during occlusion by-passed blood will be, to some extent, left there. This remaining blood lessens the difference between the impedance before and during occlusion, consequently the fluctuation of the flow curve is calculated much smaller than as it is. The mean flow in the inner layer shown in Fig. 3 also seems to be much smaller in appearance. Moir and Debra ${ }^{18}$ suggest that in the experiment in RI clearance method there may be isotope uptake of endocardial side directly from the ventricular cavity. 
According to Kinnen et al, $^{19)}$ the specific resistance of cardiac muscle and blood of a dog at body temperature and $100 \mathrm{kHz}$ are $456 \Omega \cdot \mathrm{cm}$ and $108 \Omega \cdot \mathrm{cm}(29 \%$ hematocrit) to $153 \Omega \cdot \mathrm{cm}(47 \%$ hematocrit) respectively. The results shown in Fig. 7 took generally a little larger value compared with the values calculated from the data by Kinnen et al. ${ }^{19)}$ This might be caused by the difference of frequency used in the measuring device or size and type of electrodes. The specific resistance of blood is also dependent on hematocrit and a nearly linear relationship between blood and hematocrit is obtained. In order to keep a linear relationship between $\rho_{\mathrm{b}} / \rho_{\mathrm{c}}$ and hematocrit shown in Fig. $7, \rho_{\mathrm{c}}$ should be independent of hematocrit, therefore the data suggest that the blood volume contained in the myocardium is a little.

\section{AGKnowledGements}

The author is heartly indebted to Prof. Hiroshi Sasamoto and Dr. Yoshiro Nakamura for their helpful suggestions and their valuable advice. The author also wishes to thank Drs. H. Nagoshi, M. Takahashi, H. Nakazawa, and N. Matsumura for their cooperation and many helpful discussions.

\section{References}

1. Eckstin RW, Moir TW, Driscol TE: Phasic and mean blood flow in the canine septal artery and an estimate of systolic resistance in deep myocardial vessels. Circulat Res 12: 203, 1963

2. Eckenhoff JE, Hafkenschiel JH, Harmel MH, Goodale WT, Lubin M, Bing RJ, Kety SS: Measurement of coronary blood flow by the nitrous method. Am J Physiol 152: 356, 1948

3. Love WD, Romney RB, Burch GE: Comparison of distribution of potassium and exchangeable rubidium in organs of $\mathrm{dog}$, using rubidium ${ }^{86}$. Circulat Res 2: 112, 1954

4. Griggs DM, Jr, Nakamura Y: Effect of coronary constriction on myocardial distribution of iodoantipyrine-I331. Am J Physiol 215: 1082, 1968

5. Sevelius G, Johnson PG: Myocardial blood flow determined by surface counting and ratio formula. J Lab Clin Med 54: 669, 1959

6. Nyboer J: Electrical Impedance Plethysmography. CG Thomas, Springfield, Ill, 7, 1959

7. Allison RD, Holmes EL, Nyboer J: Volumetric dynamics of respiration as measured by electrical impedance plethysmography. J Appl Physiol 19: 166, 1964

8. Herzberg RM, Rubio R, Berne RM: Coronary occlusion and embolism: effect on blood flow in adjacent arteries. Am J Physiol 210: 169, 1966

9. Kraus JD: Electromagnetics. McGraw-Hill Book Co, New York, 120, 1953

10. Gibson JG, Seligman AM, Peacock WC, Aub JC, Fine J, Evans RD: The distribution of red cells and plasma in large and minute vessels of the normal dog, determined by radioactive isotopes of iron and iodine. J Clin Invest 25: 848, 1946

11. Love WD, Tyler MD: Effect of hypoxemia and hypercapnia on regional distribution of myocardial blood flow. Am J Physiol 208: 1211, 1965

12. Moir TW, Debra DW: Effect of left ventricular hypertension, ishemia and vasoactive drugs on the myocardial distribution of coronary flow. Circulat Res 21: 65, 1967

13. Myers WW, Honig CR: Number and distribution of capillaries as determinants of myocardial oxygen tension. Am J Physiol 207: 653, 1964 
14. Johnson JR, D Palma JR: Intramyocardial pressure and its relation to aortic blood pressure. Am J Physiol 125: 234, 1939

15. Gregg DE, Eckstein RW: Measurements of intramyocardial pressure. Am J Physiol 132: 781,1941

16. Kirk ES, Honig CR: An experimental and theoretical analysis of myocardial tissue pressure. Am J Physiol 207: 361, 1964

17. Bartelstone HJ, Scherlag BJ, Cranefield PF, Hoffman BF: Partition of canine coronary blood How. Bull New York Acad Med 42: 951, 1966

18. Moir TW, Debra DW: Measurement of the endocardial distribution of left ventricular coronary blood flow by $\mathrm{Rb}^{86}$ chloride. Am Heart J 69: 795, 1965

19. Kinnen E, Kubicek W, Hill P, Turton G: Thoracic cage impedance measurements. (Tissue resistivity in vivo and transthoracic impedance at $100 \mathrm{kc} / \mathrm{s}$.) USAF School of Acrospace Medicine technical documentary report SAM-TDR-64-5, Brooks Air Force Base, Texas, 1, 1964 